\title{
EL PROGRAMA DE MAESTRÍA DE LA ESCUELA GRADUADA DE TRABAJO SOCIAL \\ DE LA UNIVERSIDAD DE PUERTO RICO: UNA EXPLORACIÓN MICROETNOGRÁFICA DE LA COMUNIDAD DE APRENDICES ${ }^{1}$
}

\section{Úrsula Manfredo Plicet ${ }^{2}$}

\section{Resumen}

Estudio para explorar cuales procesos del liderazgo educativo administrativo y didáctico- ocurren, y documentar cómo ocurren, para describir la comunidad de aprendices en el Programa de Maestría de la Escuela Graduada de Trabajo Social Beatriz Lasalle (PMTS). El estudio estuvo enmarcado en el Paradigma Dialéctico de la Administración Crítica (Rodríguez Matos, 2001), en la conceptuación de la misión de la Universidad del Siglo XXI, liderazgo educativo, y las comunidades de aprendices. El diseño fue el micro etnográfico crítico, con múltiples estrategias cualitativas para la recopilación de la información. Se analizó la información desde el modelo de Harry F. Wolcott (1994) y el programa de computadora QSR NVIVO. Los hallazgos demuestran que el liderazgo educativo está vinculado a la perspectiva paradigmática que permea la cultura organizacional, matizados por el paradigma positivista; y que la adopción de un nuevo paradigma humanista crítico es insuficiente para la transformación de la cultura organizacional. Con la adopción del Paradigma de Comunidades Universitarias de Aprendices propuesto, el PMTS tomaría distancia del paradigma positivista y entraría de una manera dinámica en sintonía con el Paradigma Humanista Crítico adoptado.

\footnotetext{
${ }^{1}$ Artículo basado en mi disertación doctoral aprobada en la Facultad de Educación área de administración y supervisón educativa de la Universidad de Puerto Rico, 2007.

2 Directora de la Oficina de Adiestramiento y Garantía de Calidad de la Administración de Familias y Niños del Departamento de la Familia.
} 
Descriptores: Liderazgo educativo (administrativo y didáctico), comunidad de aprendices, misión de la universidad del Siglo XXI y paradigma.

\section{Abstract}

The purpose of this research was to explore and document which educational leadership, administrative and professional development processes occur, in order to describe the community of learners. This study was framed in the Critical Administration Dialectic Paradigm (Rodriguez Matos, 2001), the literature on the mission of the University in the XXI century, the administrative and professional development leadership, and the community of learners. The research design was critical ethnographic. Qualitative techniques were used for data collection. Data was analyzed using Harry F. Wolcott's (1994) model and the computer program QSR NVIVO. The results demonstrated that the educational leadership is linked to the positivistic paradigmatic perspective that permeates the organizational culture. The incorporation of the critical humanistic paradigm in the MPSW, still it shows to be insufficient in order to transform the organizational culture. By adopting the Paradigm for the University Community of Apprenticeships that I propose, the MPSW would distance itself from the positivistic paradigm and would operate in tune with the adopted Critical Humanistic Paradigm.

Key words: Educational leadership (administrative and professional development), communities of learners, mission of the university of XXI Century and paradigm.

\section{Introducción}

El propósito de esta investigación fue explorar qué procesos del liderazgo educativo -administrativo y didáctico- ocurren, y documentar cómo ocurren, para describir la comunidad de aprendices en el Programa de Maestría de de la Escuela Graduada de Trabajo Social Beatriz Lassalle, de la Universidad de Puerto Rico, Recinto de Río Piedras (PMTS) a la luz del Paradigma Dialéctico de Administración Crítica de José Miguel Rodríguez Matos (2001). Para mayo del 2004, realicé un estudio etnográfico en el Programa de Maestría de Trabajo Social de la Universidad de Puerto Rico (Manfredo Plicet, 2004). Este estudio piloto surge como una fuente de inspiración para explorar y documentar los procesos en la construcción de la comunidad de aprendizaje en este programa de maestría. Su diseño fue exploratorio y 
fenomenológico. El eje del estudio fue el contexto educativo del PMTS y su postura de liderazgo educativo, en los procesos formativos de los trabajadores sociales ante las aceleradas transformaciones producto de los cambios en las relaciones sociales entre el Estado y la sociedad civil, entre lo público y privado.

Como trabajadora social con más de 25 años de experiencia, no encontré otras investigaciones, en especial de tipo cualitativo - etnográfica, que estudiaran este fenómeno. El diseño exploratorio - etnográfico, seleccionado para este estudio ofrece ventajas en cuanto al trabajo de campo para llegar a conocer a fondo el grupo social que es objeto de estudio. Para recopilar la información se llevaron a cabo entrevistas semi estructuradas a los tres ex directores de la Escuela Graduada de Trabajo Social Beatriz Lassalle, desde 1984 hasta la actual directora en su escenario cotidiano. En esta búsqueda de voces resonantes se entrevistaron a dos miembros de facultad, así como a un grupo focal de estudiantes. Además, realicé observaciones al contexto educativo, las que anoté en el diario de campo. Por otro lado, leí los autoestudios para los procesos de reacreditación ante el "Council on Social Work Education (CSWE)" correspondientes a los años 1994 y 2002. La información recopilada la analicé utilizando las teorías de educación crítica de Freire (1970) y de Guirox (1995). Se redujo la información recopilada mediante el uso del programa computadorizado QSR NVIVO.

Los hallazgos de esa investigación me permitieron esbozar los procesos de liderazgo educativo presentes en el contexto educativo en una fusión con el andamiaje que, por ende, incide en la formación de trabajadores y trabajadoras sociales. Las voces participantes, las observaciones de campo y el uso de otros instrumentos, hicieron emerger las características de los procesos de liderazgo educativo, del que se deriva el desarrollo profesional, las relaciones, los procesos de participación, el foco de enseñanza, el clima y las condiciones físicas. Por su parte, el andamiaje de acuerdo a lo observado, son los valores, la visión, la misión, el género, los paradigmas, los discursos, el contexto y texto del currículo y los resultados educativos esperados. Estos, de acuerdo a la literatura, son parte en la construcción de la comunidad de aprendices.

En mi estudio encontré que el marco que postula el contexto exterior, así como otros elementos en el contexto interno en el que opera la Escuela, tales como algunas corrientes teóricas prevalecientes y tradicionales, dado la escuela de pensamiento de la que proviene el docente y la forma de operar el 
trabajo social en las agencias de servicios humanos, era el sistémico positivista (Manfredo Plicet, 2004). Este discurso y paradigma incide en la configuración del currículo que es formador de profesionales de trabajo social. Por eso, el propósito de esa investigación fue hacer una denuncia acerca del marco teórico que es la base de modelos que guían la intervención de los trabajadores y trabajadoras sociales. Es el uso de un currículo que mantiene el 'status quo'. Es importante mencionar que la filosofia educativa y las políticas sociales que enmarcan el andamiaje de los procesos del liderazgo educativo del Programa de Maestría en Trabajo Social se vinculan al Estado Benefactor por la relación histórica política de Puerto Rico y Estados Unidos de Norte América, así como los requisitos de acreditación del Council on Social Work Education (CSWE). Esto concuerda con los hallazgos de Díaz Pérez (1978) y de Lizardi (1983).

Por su parte, en la línea de pensamiento acerca de lo universitario, lanzo el reto a la misión de la universidad propuesta por Ortega y Gasset (1936), enseñar las profesiones, conservar el conocimiento y crear y transmitir el conocimiento. La universidad moderna tiene diferentes medios para cumplir con su misión a través de la enseñanza, la investigación y la extensión. La extensión es la prolongación natural de la aplicación de la enseñanza, la extensión es la obra de la universidad puesta al servicio de la sociedad para mejorar la calidad de la vida en la comunidad (Fasoli, 2003; Malott, 1998). La educación es un instrumento para el cambio social (Morow y Torres, 2002; Tedesco, 1985). Por ende, la educación universitaria es indispensable para asegurar la transformación social y la formación de ciudadanos capaces de enfrentar los desafíos del siglo XXI, entre los cuales se encuentra la globalización.

En el contexto puertorriqueño la Universidad de Puerto Rico, Recinto de Río Piedras, es la primera en ser fundada en el año 1903. Se reconoce la misión del recinto de Río Piedras como una de servicio a la sociedad. Esta misión de servicio a la sociedad se refleja en la Universidad de Puerto Rico, Recinto de Río Piedras, mediante el documento: Hacia una comunidad de aprendizaje: Reflexiones y recomendaciones en torno a los procesos de enseñanza aprendizaje en el Recinto de Río Piedras (1999). En segundo lugar, la Escuela Graduada de Trabajo Social Beatriz Lassalle de la Universidad de Puerto Rico, Recinto de Río Piedras, su origen se remonta al año 1929. Se le atribuye la más antigua tradición en la formación de los profesionales de trabajo social en Puerto Rico (Díaz Pérez, 1978; Lizardi, 1983), de quienes se espera contribuyan en mejorar la calidad de vida de la 
sociedad. En armonía con esto, es conveniente esbozar que al igual que Derrida (1984), propongo la 'problematización' de la universidad. Cuando escribe que la universidad no se compone sólo de muros y estructuras exteriores que la rodean y protegen nuestro trabajo, sino que también es la estructura de nuestra interpretación. Mi interpretación es que la educación es un instrumento para el cambio social, la que no debe verse como instrumento de garantía para la reproducción cultural o para institucionalizar el cambio o la innovación para perpetuar precisamente las estructuras predominantes del Estado. Desde el punto de vista de la sociología de las profesiones, las universidades producen y administran las formas de conocimiento socialmente aceptadas y asociadas a las prácticas de ciertas profesiones (Dingwall y Lewis, 1983).

Por lo tanto, estas razones contextuales y el reclamo por cambios con los estilos tradicionales en el liderazgo educativo, dan paso a explorar otros paradigmas emergentes que se acoplen a la complejidad humana en la educación como las Comunidades de Aprendices. En la literatura varios autores han abordado el tema con especial interés. Estos autores plantean que en las comunidades de aprendices los educadores trabajan y colaboran entre sí, mejora la calidad del proceso de enseñanza aprendizaje en la organización (McLaughlin y Talbert 2001; Michell y Sackney, 2000), mejora la capacidad individual y colectiva para el proceso de enseñanza aprendizaje (Elmore, 1995; Newman y Asociados, 1996). Además, la noción de comunidad ofrece la oportunidad de desarrollar el potencial en los miembros internos y externos de la organización educativa para que aprendan unos de otros. De igual modo, se promueve la identificación colectiva de estrategias para la solución de problemas tales como el reclutamiento y la retención del personal. Por esta razón, aumenta el fortalecimiento del repertorio de respuestas para lidiar con los problemas complejos (Toole y Louis, 2002).

Por su parte, según Hipp y otros (2003), desde que Peter Senge publicó en 1990 La quinta disciplina, tanto los miembros del mundo corporativo como los educadores se han esforzado por adoptar y mantener las comunidades de aprendices como una promesa para la reforma organizacional. De manera muy limitada se han realizado investigaciones acerca de cómo esas comunidades de aprendices se perfilan en realidad (Darling-Hammond, 1996; DuFour y Eaker, 1998; Fullan, 2000; Senge, 1990). En forma similar, son muy escasos los trabajos a nivel universitario. De ahí que mi propuesta va dirigida hacia las Comunidades Universitarias de Aprendices, un 
paradigma emergente en el liderazgo educativo. Surge como un cuerpo articulado de conocimientos.

Desde la dimensión del liderazgo administrativo se sustenta en el Paradigma Dialéctico de la Administración Crítica propuesto por Rodríguez Matos (2001). Este concepto es eje en esta investigación, dado el enfoque de criticidad con el que fue valorado el fenómeno estudiado. Por lo que corresponde plantear que en el Paradigma Dialéctico de la Administración Crítica, se conjugan premisas como la responsabilidad compartida y la administración educativa como proceso dinámico en constante cambio. Este enfoque, además, propicia que la organización educativa se relacione con el mundo exterior en constante evolución y con el contexto socio- histórico en que opera la organización educativa.

Otras posturas teóricas que sustentan el Paradigma de las Comunidades Universitarias de Aprendices del liderazgo educativo son el movimiento moderno del aprendizaje organizacional, propuesto por Senge (1990) y Argyrys y Schön (1996) y la estrategia de la administración del cambio de las organizaciones educativas, que vinculamos con la propuesta de Drucker (1997), así como el liderazgo trasformador postulado por Burn (1978), Roueche, Baker y Rose (1989) según citados en el estudio de análisis metaetnográfico de Pielstick (1998), quienes centran el enfoque en el liderazgo en el ámbito educativo e identifican la importancia de la visión compartida entre otros factores en el liderazgo transformador. Desde la dimensión del liderazgo didáctico, otras posturas abonan al Paradigma emergente de las Comunidades Universitarias de Aprendices, las vemos representadas por Knowles (1980), Ramos (1999) y Sparks y Hirsh (1997). Los aspectos normativos en este paradigma se ve reflejado en el Informe de la UNESCO de la Comisión Internacional sobre la Educación para el Siglo XXI (1996) y con la Cátedra de la UNESCO de Gestión, Innovación y Colaboración en la Educación Superior.

Esta investigación se propone abonar al cuerpo de conocimientos que sustenta las Comunidades Universitarias de Aprendices, paradigma emergente propuesto por mí, en el liderazgo educativo. Este paradigma en el liderazgo educativo, se acopla a la complejidad humana en la educación y promueve alcanzar los fines de la educación universitaria. Es por ello, que esta investigación centra su foco en la exploración del liderazgo educativo para documentar los procesos, para contribuir en lograr los fines inmediatos de la educación, de acuerdo al Paradigma Dialéctico de la Administración 
Crítica (Rodríguez Matos, 2001), mediante una comunidad de aprendices en el Programa de Maestría de la Escuela Graduada de Trabajo Social de la Universidad de Puerto Rico.

\section{Descripción del problema}

El PMTS se ve impactado por los procesos de cambios acelerados y la globalización. Por la repercusión de estos procesos en la manera tradicional y burocrática de administrar y cómo operan los procesos en el contexto educativo, así como por las limitaciones que surgen para explicar las contradicciones y complejidades del liderazgo educativo, el que relacionamos con el mundo exterior en constante cambio. Como se ha mencionado, la relación histórica política de Puerto Rico y Estados Unidos de Norte América, así como los requisitos de acreditación del CSWE plantean la necesidad de explorar otros paradigmas emergentes en liderazgo educativo, como las Comunidades Universitarias de Aprendices. Este es un paradigma emergente en el lidérazgo educativo, que permite articular prácticas eminentemente participativas y de cambio con los estilos tradicionales de administrar y desarrollar los recursos humanos que participan en los procesos educativos y relacionado al contexto educativo en el que opera (Rodríguez Matos, 2001).

Desde la misión de la universidad del Siglo XXI, la profesión de trabajo social al igual que otras, en el concierto de las profesiones, incluye creación de conocimiento, desarrollo de la responsabilidad social y el servicio a la sociedad. El PMTS tiene la más antigua tradición en la formación de trabajadores y trabajadoras sociales en Puerto Rico, en aras de "desarrollar profesionales que trabajen con individuos, familias, grupos y comunidades, o en la administración de servicios que contribuyan en el desarrollo de políticas, programas y servicios para una mejor sociedad" (Universidad de Puerto Rico, 2002). No obstante, la prensa diaria del país destaca el hecho de las grandes crisis personales, familiares, comunitarias e institucionales a las que se enfrenta nuestra ciudadanía y la situación en que se encuentran los servicios a la familia, a los estudiantes en las escuelas, entre otros. Como señala Malacalza (2003), la sociedad se transforma radicalmente, producto del interjuego de las relaciones sociales, por la relación entre el Estado y la sociedad civil. Señala esta autora que esto coloca en una posición de vulnerabilidad a las unidades académicas de trabajo social, que es necesario 
analizar profundamente para generar cambios necesarios y revertir los posibles resultados adversos por la falta de acción.

Desde los argumentos anteriores, solamente encontré dos estudios acerca de la Escuela Graduada de Trabajo Social Beatriz Lassalle de la Universidad de Puerto Rico, realizados por Díaz Pérez (1978) yl Lizardi (1983). Estas investigaciones no aluden a mi tema de estudio por lo que abonan en mi argumentación que en Puerto Rico no se han documentado las observaciones acerca de la investigación del tema y la correspondiente valoración desde el marco teórico que he esbozado.

\section{Breve revisión de literatura}

Este estudio se ancla en el Paradigma Dialéctico de la Administración Crítica, (Rodríguez Matos, 2001) porque de acuerdo a este autor, la administración hay que relacionarla con el mundo exterior en constante revolución. Es la práctica crítica individual y grupal que integra las tareas y los procesos con la teoría y con el contexto socio- histórico en que opera la organización educativa. Tiene como punto de partida la cultura organizacional que impacta, a la vez que responde, a las fuerzas internas y externas que inciden en dicha organización.

Desde el concepto del liderazgo educativo, es pertinente recalcar que "sus fines inmediatos son: el desarrollo integral del educando, el desarrollo integral de los recursos humanos, la viabilización de los propósitos organizacionales y el desarrollo, la integración o la transformación de la organización según las demandas del momento histórico. Opera en relación dialéctica con los aspectos que la sociedad necesita preservar, integrar o transformar." (Rodríguez Matos, p.50). Entonces, ¿cómo conciliamos este panorama de cambios y los fines de la educación aquí planteados? Surge entonces mi planteamiento de la ruptura paradigmática en el liderazgo educativo.

Para abordar el tema de la ruptura paradigmática es muy útil la argumentación que esboza el Paradigma Dialéctico de la Administración Crítica que ha conceptuado Rodríguez Matos (2001), inspirado en la pedagogía crítica de Paulo Freire. Presenta el paradigma crítico como una alternativa frente al paradigma empírico analítico. Por otra parte, este paradigma "permite adentrarse en las fuentes "problematizadoras" de las 
interacciones sociales y de los conflictos que tienen lugar en el proceso administrativo y sus múltiples contradicciones" (p.143).

Con el propósito de articular y argumentar acerca del paradigma emergente de las comunidades universitarias de aprendices en el liderazgo educativo, es preciso resumir algunas de las premisas que sustentan el Paradigma Dialéctico de la Administración Crítica (Rodríguez Matos, 2001). Estas premisas son: la administración es compartida, el proceso administrativo es esencialmente cambiante, todas las consideraciones particulares como globales son claves en el proceso decisional, el fin inmediato del proceso administrativo educativo es multidimensional, el proceso administrativo local es parte del proyecto nacional global, los paradigmas tradicionales no contemplan la realidad social, cultural, política y económica que operan las organizaciones educativas. Es conveniente insertar en esta exposición que el Paradigma Dialéctico de la Administración Crítica fue utilizado por Morales Santiago (2003), para abordar su tema de disertación doctoral titulada: Hacia una concepción del liderazgo crítico en la administración educativa. Se estudió una posible concepción del liderazgo crítico en la administración educativa. Los hallazgos establecieron que se toma en consideración el contexto social como referente y es un todo dinámico y dialéctico entre la teoría y la praxis (p.v). Por su parte, Castillo Ortiz (2002) reseñó mediante una prospectiva crítica del libro de la Estructura de la administración crítica: Una interpretación dialéctica del doctor José Miguel Rodríguez lo siguiente:

El doctor Rodríguez presenta un paradigma dialéctico de la administración educativa en el contexto de la realidad histórica y social puertorriqueña. Este es un modelo flexible y multidimensional, dinámico e interactivo con un acercamiento humanista a la administración educativa como campo dinámico y complejo. El mismo va orientado a proveer un marco teórico coherente que de cohesión a la totalidad de la vida organizacional y que provea a su vez una lógica para interpretar y guiar la práctica administrativa educativa (p.104).

Por su parte, otros conceptos que abonaron en sustentar esta investigación son los atributos de las comunidades de aprendices. Un atributo común en todas las definiciones acerca de las comunidades de aprendices implica que se trata de un acto de participación caracterizado por un sentido o propósito común, una misión, visión y valores compartidos. 
En las comunidades de aprendices todos aportan, reflexionan y coconstruyen el conocimiento. Se trata de una comunidad humana que se construye y se involucra en un proyecto educativo y cultural propio, con el propósito de educar a sus constituyentes en el marco de un esfuerzo endógeno, cooperativo y solidario (García Fernández, 2002; RojasDrumont, 1998; Villalobos Torres, 2000). Según Bryk y Driscroll (1988), los atributos de las comunidades de aprendices son: un sistema de creencias y valores compartidos, actividades comunes que vinculan a unos miembros con otros, con la educación en general y con las tradiciones del contexto educativo. Para Raywid (1993), los atributos de las comunidades de aprendices son respeto, cuidado, inclusión, confianza, apotestamiento y compromiso con sus actividades académicas. Royal y Rossi (1997), en colaboración con John Gardner de la Universidad de Standford, refinaron estos conceptos. Según estos autores, en las comunidades de aprendices la comunicación es abierta, la participación es ampliamente practicada, el trabajo en equipo prevalece y el concepto de diversidad es incorporado. Los miembros del personal y los aprendices comparten una visión común acerca de la educación del futuro y reconocen los esfuerzos y logros de los demás.

Estos atributos son útiles para relacionarlos con la perspectiva de liderazgo educativo y los contextos educativos como organizaciones que aprenden. Por lo tanto, es conveniente insertar el concepto de la organización de aprendizaje, para la creación de contextos educativos como organizaciones que aprenden. De acuerdo con Senge (1990), es una organización capaz de expandir su capacidad en forma continua para crear su futuro. Una expansión y un futuro que relaciono con los propósitos de la educación universitaria, ámbito de este estudio.

Congruente con el propósito que guió esta investigación, desarrollé una microetnografía crítica de la educación en el PMTS.

\section{Preguntas de investigación}

¿Qué procesos del liderazgo educativo (administrativo y didáctico) se observan en la comunidad de aprendices en el Programa de Maestría de Trabajo Social de la Universidad de Puerto Rico, Recinto de Río Piedras? 
¿Cómo ocurren los procesos del liderazgo educativo (administrativo y didáctico) en la comunidad de aprendices en el Programa de Maestría de Trabajo Social de la Universidad de Puerto Rico, Recinto de Río Piedras?

\section{Delimitaciones y limitaciones}

Este estudio se delimita a investigar el liderazgo educativo desde las dimensiones del liderazgo administrativo y del liderazgo didáctico en el nivel universitario, el PMTS. Además, se limita a investigar como punto de partida a nivel documental desde el año 2002 hasta el 2007, dado que el año 2002, marca el periodo del último informe de Autoestudio para la reacreditación por el CSWE del PMTS. Además este estudio estuvo limitado a desarrollarse durante 22 semanas, entre 2006-2007.

\section{Enfoque metodológico}

Estudio microetnográfico, exploratorio y fenomenológico en el PMTS. Está anclado en la teoría crítica, la cual ha marcado una notable influencia en el desarrollo de las investigaciones cualitativas críticas en el campo de la educación en general (Kinchoele y McLaren, 2002; Lucca Irizarry y Berríos Rivera, 2003; Solís Jordán, 2005).

Cuando utilizamos esta metodología, los participantes se expresan de manera oral o escrita y el comportamiento de éstos es observado por el investigador. Las observaciones se realizan en el escenario natural (Lucca Irizarry y Berríos Rivera, 2003). Por su parte, Solís Jordán (2005) plantea que en la investigación cualitativa se describe el fenómeno o fenómenos "con el propósito de proyectar un compromiso con el cambio social y el desarrollo de la educación como expresión social" (p.11). Con el desarrollo de esta investigación queda plasmado mi compromiso con la educación pública en trabajo social en Puerto Rico y con el liderazgo educativo que lo guía. La propuesta de las comunidades universitarias de aprendices es un paradigma del liderazgo educativo que se perfila más armónico con los cambios acelerados y los desafíos que plantean estos cambios, particularmente en la educación universitaria. 


\section{Diseño del estudio}

El diseño metodológico fue la microetnografía crítica. El etnógrafo no segmenta las experiencias que estudia en variables, las examina como un todo integrado. Esto es cónsono con el planteamiento del paradigma crítico. Por ejemplo, en cuanto a la metodología, en el paradigma crítico se plantea que la realidad está moldeada por el contexto socio-histórico, evoluciona con el tiempo, es dialógico e inductivo.

Por lo tanto, desde la perspectiva del liderazgo educativo, Rodríguez Matos (2001) coincide en torno a investigar desde el paradigma de la teoría crítica. Los temas idóneos para la investigación que resaltan son los procesos de la administración educativa, el examen de las prácticas vinculadas a la toma de decisiones, la responsabilidad compartida y los estilos no tradicionales de administrar o ejercer el liderazgo. Por ende, quiero resaltar que no cabe duda que el problema propuesto se presta para ser estudiado desde el diseño de la microetnografía crítica de la educación.

En el desarrollo de este estudio, utilicé de referencia de manera amplia y flexible los pasos en el desarrollo de un estudio etnográfico que presentan Lucca Irizarry y Berríos Rivera (2003) y las fases contempladas por Carspecken (1996) en su modelo para la etnografía crítica. En forma similar, estos autores proponen cinco etapas que a continuación describo para ilustrar cómo fluyeron en el desarrollo del estudio. La primera etapa es la de cuajar el problema a ser investigado y crear las preguntas de investigación. La segunda etapa incluyó la observación y la entrada en forma gradual al PMTS, la identificación y la entrevista de los informantes clave que grabé en cintas de audio así como la anotación en el diario de campo. Una vez lograda la entrada en forma gradual, entré en la tercera etapa, o de calentamiento en la cual la comunicación fluyó con facilidad y me acerqué más al fenómeno de estudio. Aquí surgieron nuevos ángulos e interrogantes que me ayudaron refinar aún más el problema de estudio. En la cuarta etapa o de trabajo intenso visité con mayor comodidad el PMTS, entrevisté a los participantes de los grupos focales que grabé en cintas de audio. Asimismo, puse en marcha una interpretación de las características de la cultura estudiada, desde la perspectiva del Paradigma Dialéctico de Administración Crítica y del marco teórico adoptado, en la creación de una comunidad universitaria de aprendices en el PMTS. Las observaciones de campo que realicé las anoté en el diario de campo. Tras haber hecho acopio de la 
suficiente información para contestar las preguntas del estudio, inicié la quinta etapa o la retirada en forma gradual.

\section{Participantes del estudio}

Los participantes ${ }^{3}$ fueron las voces del contexto. Representados por los miembros de facultad, los estudiantes y del personal administrativo y de la biblioteca sobre el tema de la investigación, acerca de los procesos del liderazgo educativo que ocurren y cómo ocurren para describir la comunidad de aprendices en el PMTS. Lo que me permitió conocer por separado sus opiniones. Para propósitos de este estudio los grupos focales se constituyeron con un total de 13 participantes, con la finalidad de mantener el propósito explícito de interacción de las opiniones de los participantes.

\section{Estrategias y medios para recopilar la información}

- Entrevistas

- semi estructuradas a cinco informantes clave

- tres grupos focales

- Observaciones libres

- Guía de análisis del contenido de los documentos

- Diario de campo para tomar notas

- Grabación en audio y transcripción de las entrevistas

\section{Análisis de la información}

La información recopilada, fue leídas varias veces. Esto para que haga sentido las voces y los objetos en los cuales se coloca mayor interés (Geertz, 1980, p.167, citado en Thomas 1993).

En la investigación etnográfica crítica, la interpretación cobra un significado de relieve. Es algo más que una atractiva lista de tipologías y etiquetas "que haga sentido a nosotros" pues requiere prestar atención a las instituciones, las acciones, las imágenes, los eventos, las costumbres y todos los objetos de interés científico, así como los objetos en los cuales se coloca mayor interés (Geertz, 1980, p.167, citado en Thomas 1993).

${ }^{3}$ En el trabajo utilizo el término participantes para hacer referencia tanto al masculino como al femenino, para evitar distracciones durante la lectura. 
La información recopilada la organicé en forma sistemática, con la finalidad de analizar y establecer categorías y relaciones con las preguntas de investigación de una manera inductiva, lo que me permitió ir descubriendo el fenómeno estudiado. La codificación la realicé con el programa de computadora para análisis de datos cualitativos QSR NVIVO (2002). La información de este estudio la analicé con el modelo de Harry F. Wocott (1994) que consta de tres componentes principales: la descripción, el análisis y la interpretación (Lucca Irizarry y Berríos Rivera, 2003). Parte integral del proceso de análisis es la triangulación. La misma la realicé con la información recopilada de las fuentes, es decir, los informantes clave, los grupos focales y mis observaciones como investigadora anotadas en el diario de campo. Además, la comparé con el resultado de la lectura de documentos, todo esto teniendo como referente las preguntas de investigación.

\section{Hallazgos}

Los hallazgos de este estudio, se presentaron con la utilización de la narrativa en el siguiente orden: en primer lugar, la cultura organizacional del PMTS. En segundo lugar, perfilo las prácticas administrativas y su relación con el contexto interior y exterior en que opera el PMTS. En tercer lugar, esbozo el liderazgo educativo (administrativo y didáctico) con respecto a qué procesos ocurren y cómo ocurren.

Esta estructura es cónsona con los propósitos de este estudio y está en armonía con los postulados del Paradigma Dialéctico de la Administración Crítica. El Paradigma establece que la cultura organizacional es el punto de partida e impacta, a la vez que responde, a las fuerzas internas y externas que inciden en las organizaciones. De igual modo, esto es congruente con el diseño micro etnográfico crítico de este estudio.

Por otro lado, señalo que las dimensiones del liderazgo educativo (administrativo y didáctico) no se dan en forma aislada sino que actúan en forma dinámica y simultánea. En la narrativa expongo simultáneamente la descripción, el análisis y la interpretación de la exploración realizada de acuerdo a las preguntas de investigación que guiaron este estudio (Lucca Irizarry y Berríos Rivera, 2003). La narrativa la fundamenté con pasajes o extractos de los testimonios de los participantes, las transcripciones de los 
pasajes del diario de campo y de la lectura de documentos, en adhesión a los marcos teóricos. También, expresé mi voz desde lo que observé como investigadora.

\section{La cultura organizacional}

El número de la matrícula del PMTS es de 172 estudiantes, en los programas diurno y vespertino y sabatino y predominan las mujeres. El número de profesores es de 20 a tiempo completo, de éstos 17 con grado doctoral y tres con cursos conducentes al doctorado y 10 por contrato. En la Facultad también predominan las mujeres. El PMTS está organizado en tres concentraciones a saber: Familia, Comunidad y Administración de Servicios Humanos. Diez empleados administrativos comparten funciones del PMTS y el Programa Doctoral. Los empleados administrativos ocupan posiciones diversas como secretaria administrativa, oficial administrativo, secretarias, recepcionista y oficial de reproducción de material audio visual. Estos datos ofrecen la dimensión tradicional de la cultura organizacional.

Por su parte, desde el factor humano que nos recalca Rodríguez Matos (2001) en el Paradigma Dialéctico de la Administración Crítica, hace falta añadir voces representativas de lo que expresaron los participantes. Por lo tanto, los pasajes de mi estudio me lo contaron los docentes, los estudiantes y el personal administrativo y de la biblioteca que me abrieron el camino para entrar al PMTS y me acompañaron en esta travesía requisito indispensable en los estudios etnográficos.

La cultura organizacional está matizada por lo temporal y lo espacial, elementos propios del contexto estudiado. Lo temporal se refiere al tiempo que impone los períodos del calendario académico y la duración del programa de estudios que determina la estadía de los estudiantes que forman parte de la comunidad de aprendices; mientras que lo espacial se refiere a lo que delimita el espacio físico en el que ubica el PMTS. Esto cobra significancia cuando esbozo lo plantado por Carspecken (2002) en cuanto a que los actores continuamente producen innovaciones culturales, pero están supeditadas al medio preestablecido. En otras palabras, para los fines de este estudio esto significa que lo espacial y lo físico condicionan la cultura organizacional. Esto me lleva a establecer vínculo con la definición propuesta por Rodríguez Matos (2001) con respecto a la cultura organizacional, en el sentido de que es el punto de partida e impacta, a la vez que responde, a las fuerzas internas y externas que inciden en las 
organizaciones. El siguiente pasaje de la voz de uno de los docentes ilustra esta afirmación:

Yo creo que la cultura organizacional de nuestro Programa, no se diferencia del resto de la cultura organizacional de la Institución de la Universidad de Puerto Rico. Yo creo que la reproduce en gran medida. Eh... al ser una cultura burocrática, altamente burocrática, muy especializada, jerárquica y... quizás, con unos intentos de participación de parte de cada uno de los actores. Desde su propia, su propia iniciativa, esfuerzo que hacen.

Esto apunta a que lo institucional está matizado por el paradigma clásico que permea en la Universidad de Puerto Rico.

En la Figura 1 presento una esquematización gráfica de los hallazgos con respecto a la cultura organizacional en el PMTS. 
Figura 1

Representación esquematizada de la cultura organizacional de la comunidad de aprendices del PMTS

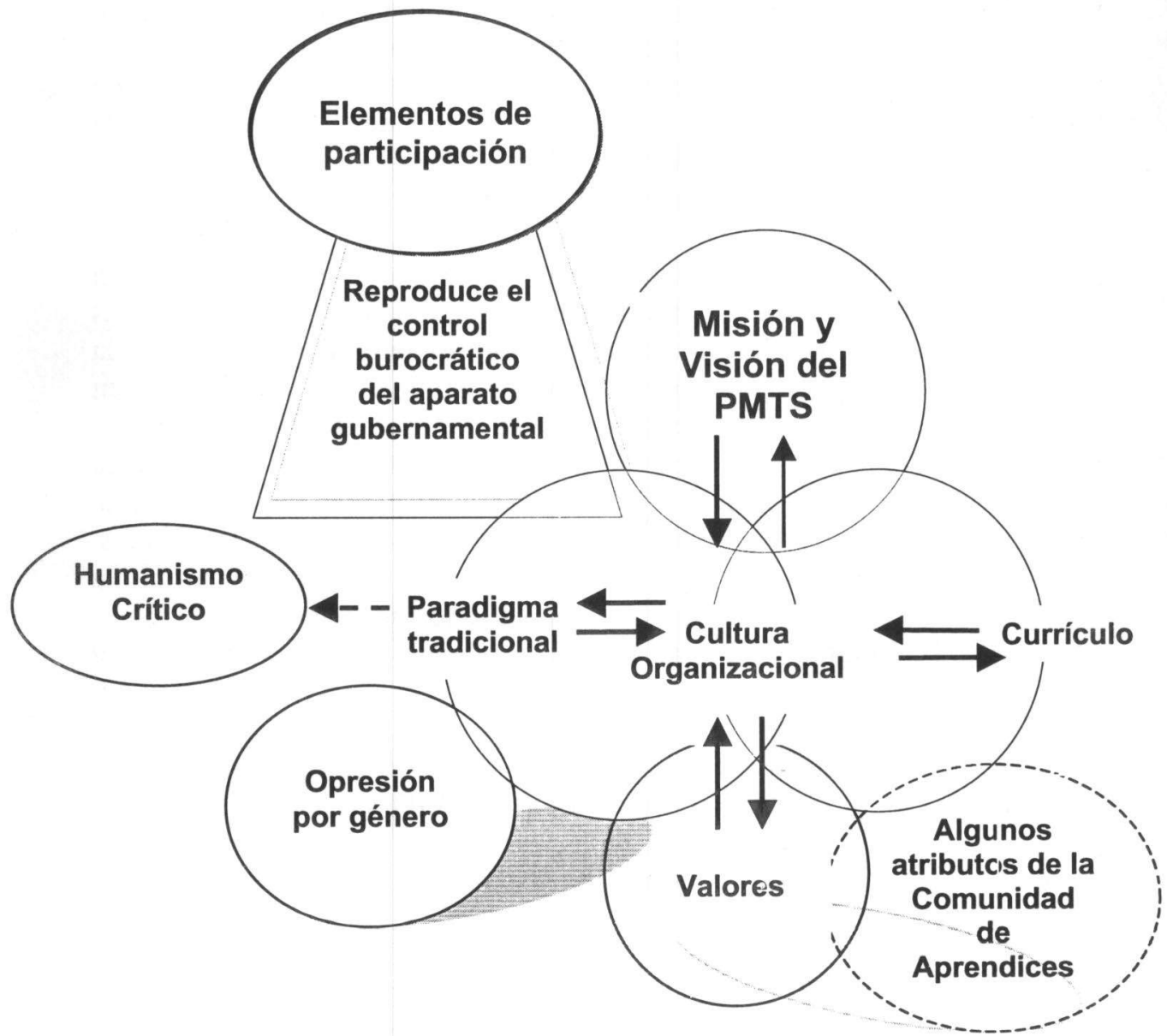


En la figura que antecede represento los hallazgos con referencia a la cultura organizacional. Desde el Paradigma Dialéctico de la Administración Crítica la cultura organizacional es el punto de partida que impacta, a la vez que responde, a las fuerzas internas y externas que inciden en dicha organización.

En primer lugar está la base de una pirámide. La misma representa la cultura organizacional burocrática con un enlace de elementos de participación en los limitados recursos fiscales que le asigna el aparato gubernamental y la búsqueda de espacios de participación para allegar otros fondos por parte de los miembros del PMTS. Esto apunta a responder las preguntas de investigación: ¿qué procesos ocurren y cómo ocurren? La burocracia es una práctica vinculada a los paradigmas tradicionales de administrar.

Este hallazgo corresponde al liderazgo administrativo. Por ende, aparece un círculo que refleja el paradigma positivista vinculado a los paradigmas tradicionales de administrar, que permea la cultura organizacional. Surge la denuncia de la opresión por género, con un óvalo. El paradigma está en transición hacia el humanismo crítico. La Misión del PMTS recibe la influencia del paradigma corresponde e impacta tanto al liderazgo administrativo como didáctico en un proceso dinámico. A su vez, la revisión de la Misión y Visión del PMTS, impacta el currículo y los valores. Todo esto en un halo, como un centro impacta y responde a la cultura organizacional del PMTS. Finalmente, inserto un óvalo con algunos atributos de la comunidad de aprendices. La presencia de estos atributos en el PMTS es débil.

\section{Las prácticas administrativas y su relación con el contexto interior y exterior en que opera el PMTS}

El siguiente pasaje de la voz de un docente ejemplariza la relación con el contexto interior.

...una de las cosas que decidimos como Facultad fue cada concentración va a desarrollar un taller para el resto de la Facultad porque yo puedo tener el conocimiento actualizado sobre teorías y prácticas sobre el área de comunidad, pero otros compañeros que están más insertos en la práctica de lo que es individuos y familia tal vez no estén al tanto de la evolución del conocimiento en esa área, entonces lo mismo 
van a hacer ellos de familia para toda la facultad y los de administración y supervisión para toda la Facultad...

En la Figura 2 presento una esquematización gráfica de los hallazgos en torno al perfil de las relaciones con el contexto interior y exterior.

\section{Figura 2}

Representación esquematizada de la cultura organizacional y el correspondiente vínculo con el contexto interior, exterior e internacional en el que opera el PMTS

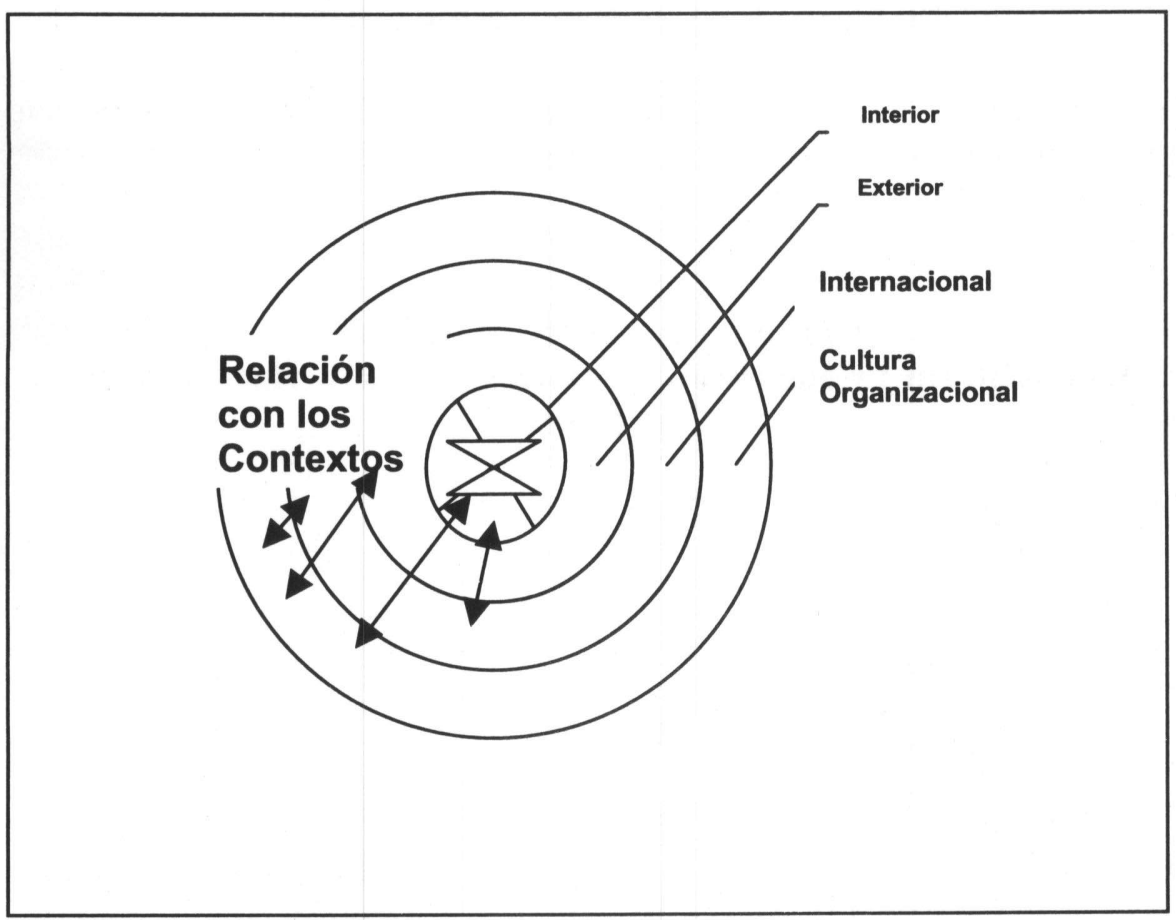

El círculo del núcleo o del contexto interior representa la Facultad especializada por concentración, refleja departamentalización y división de tareas. Las prácticas actuales están alejadas de la propuesta del Paradigma Dialéctico de la Administración Crítica y de los atributos de la comunidad de aprendices. El siguiente círculo representa la relación con el contexto en la gestión de proyectos especiales con agencias gubernamentales y las organizaciones no gubernamentales (ONG's). Estas iniciativas nutren el 
currículo y son una ventana concreta al mundo exterior en el que opera, desde una perspectiva local para impactar el proyecto nacional y luego el global. Esto está en armonía con mi interpretación de lo que establece el Paradigma Dialéctico de la Administración Crítica. El siguiente círculo, representa los vínculos con lo internacional, que en el hallazgo corresponde a Latinoamérica. Estos vínculos en cuanto a la búsqueda de alternativas para la producción de conocimiento con el paradigma humanista crítico adoptado por el PMTS. El último círculo representa la cultura organizacional. La misma se impacta e impacta las relaciones con los contextos.

\section{El liderazgo educativo (administrativo y didáctico) con respecto a qué procesos ocurren y cómo ocurren}

En la Figura 3 presento una esquematización del liderazgo educativo (administrativo y didáctico) y los procesos que caracterizan el PMTS.

\section{Figura 3}

Representación esquematizada del liderazgo educativo (administrativo y didáctico) y el correspondiente vínculo con los procesos

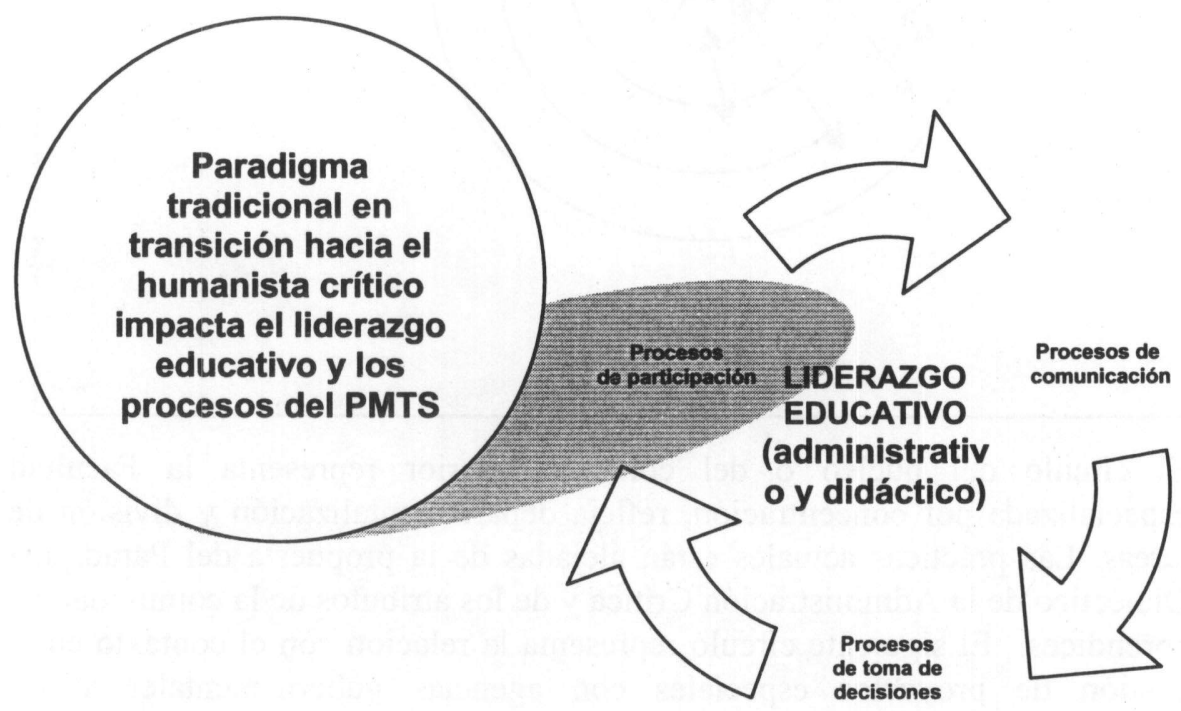


En la figura que antecede el liderazgo educativo (administrativo y didáctico) se observa impactado con la sombra del Paradigma tradicional. La transición del Paradigma tradicional al humanista crítico, ha creado una discontinuidad entre la misión y la visión y las prácticas del liderazgo. Este cambio paradigmático apunta a contestar las preguntas de investigación con respecto al liderazgo administrativo, en el sentido que la misión cambió, no obstante, las prácticas del liderazgo educativo son las tradicionales y no son cónsonas con el nuevo paradigma adoptado. Para ilustrar este hallazgo están las prácticas de enseñanza aprendizaje, el uso de la tecnología y la limitada presencia de los atributos de la comunidad de aprendices, entre los cuales se destacan el apoyo y reconocimiento para los estudiantes en algunos procesos, la práctica de escuchar y la tarea de aprender a lidiar con los conflictos. El Paradigma tradicional está muy arraigado por lo que continúa permeando los procesos de toma de decisiones, de participación y de comunicación.

En síntesis, la comunidad de aprendices del PMTS en relación al liderazgo educativo que observé presentó los siguientes atributos: ofrece reconocimiento por los compromisos académicos, apostestamiento para los estudiantes, hay trabajo en equipo por parte de la Facultad, tienen una fuerte base valorativa y respeto por la dignidad del ser humano. En torno a esto gira la convicción de aportar positivamente a la sociedad puertorriqueña.

Los procesos de participación, toma de decisiones y comunicación están mediatizados por el paradigma que permea, el positivista. Sin embargo, hay unos matices de participación en los procesos desde los intentos particulares de los actores en el contexto estudiado. Es menester reconocer que emergen unos esfuerzos por adelantar la transformación por parte de la Facultad del PMTS. Sin embargo, la participación del estudiantado y del personal administrativo y de la biblioteca debe reenfocarse.

Esto me lleva a concluir que la realidad del PMTS se aleja del Paradigma Dialéctico de la Administración Crítica en el sentido del desarrollo integral de los miembros de la organización educativa. Por otra parte, converge con la necesidad de desarrollar la Facultad en competencias de enseñar a aprender y aprender a enseñar. 


\section{Discusión y recomendaciones}

El propósito de este estudio fue explorar qué procesos del liderazgo educativo -administrativo y didáctico- ocurren, y documentar cómo ocurren, para describir la comunidad de aprendices en el PMTS, con la finalidad de identificar cuán cerca o alejado está el PMTS del Paradigma Dialéctico de la Administración Crítica, propuesto por Rodríguez Matos (2001) y de otros marcos teóricos aludidos. En este capítulo me propongo presentar la discusión de los hallazgos a la luz de la literatura revisada y estudios similares, las recomendaciones y lo que la investigación ha hecho de mí.

Los hallazgos obtenidos de esta investigación, revelan que las preguntas de investigación fueron contestadas. La información obtenida de los participantes, de mi diario de campo y de la lectura de documentos claramente aludían a unos matices de atributos de la comunidad universitaria de aprendices, acuñada por mí. Este argumento se sustenta con los propósitos compartidos durante los procesos de reacreditación y de revisión curricular, para mantener los estándares de reacreditación de manera consecutiva desde 1947 (Propuesta para la revisión del Programa de Maestría en Trabajo Social de la Facultad de Ciencias Sociales de Recinto de Río Piedras de la Universidad de Puerto Rico, 2005).

Esto demuestra el trabajo en equipo y la tradición y el orgullo institucional del que me hablaron todas las voces. No obstante, el paradigma positivista, arraigado por tantos años permea los procesos del liderazgo educativo, como la participación, la toma de decisiones y la comunicación, así como en otros procesos tales como la acreditación, la revisión curricular, la práctica supervisada, la tesis, entre otros, que emergieron durante la investigación.

La adopción del paradigma humanista crítico se observa alejado de las prácticas de liderazgo didáctico representado por autores como Knowles (1980), Ramos (1999), Sparks y Hirsh (1997). De igual modo, con los aspectos normativos en el Paradigma emergente de las Comunidades de Aprendices Universitarias que se sustenta por en el Informe de la UNESCO de la Comisión Internacional sobre la Educación para el Siglo XXI (1996). En otras palabras, el desarrollo epistemológico debe ir a la par con los desarrollos en liderazgo didáctico. 
La facultad ha dado pasos firmes hacia la adopción de un nuevo paradigma, el humanista crítico. Esto en un compromiso serio de hacer transformaciones en el renglón epistemológico del PMTS. Esto converge con lo propuesto por el movimiento moderno del aprendizaje organizacional, propuesto por Senge (1990) y Argyrys y Schön (1996), y la estrategia de la administración del cambio de las organizaciones educativas, que vinculamos con la propuesta de Drucker (1997). Este cambio paradigmático también me lleva a relacionarlo con el liderazgo trasformador postulado por Burn (1978), Roueche, Baker y Rose (1989) según citados en el estudio de análisis metaetnográfico de Pielstick (1998), quienes centran el enfoque en el liderazgo en el ámbito educativo e identifican la importancia de la visión y misión compartida entre otros factores en el liderazgo transformador. Dado que hubo consenso en las expresiones acerca de la misión del PMTS en el sentido de aportar a la sociedad puertorriqueña, converge con la misión de la universidad moderna de la aplicación de la enseñanza, la extensión que significa, la obra de la universidad puesta al servicio de la sociedad, para mejorar la calidad de la vida en la comunidad (Fasoli, 2003; Malott, 1998).

Estos procesos de cambio ocurren de manera dinámica en el PMTS, sin embargo, el paradigma de administración que permea en las estructuras del Recinto de Río Piedras es el positivista, representado por la burocracia clásica que se reproduce en el PMTS. Esto justifica mis argumentaciones en torno a la ruptura paradigmática de los paradigmas tradicionales según propuesto por Rodríguez Matos (2001), así como al señalamiento de desarrollar iniciativas endógenas culturales, propias, en adhesión a los atributos que caracterizan las comunidades de aprendices.

Otro señalamiento es la opresión por género y las relaciones asimétricas en los salones de clase. Esto responde a las visiones que permean por el paradigma positivista que se encuentra tan arraigado en lo cotidiano de la cultura observada.

La instrucción práctica y de tesis son fuente de producción de conocimiento. En la actualidad esto ocurre de manera tradicional. Es conveniente inyectarle creatividad e innovación. Sería conveniente una revisión de los métodos, para incorporar unos más activos, orientados a relacionar con mayor profundidad la teoría y la práctica (Dempsey y otros 2001). Con respecto a las destrezas del aprendizaje reflexivo de los estudiantes de trabajo social, se debe ubicar en contexto la teoría con las experiencias vividas en el día a día para atribuirles significado y vincularlas con las experiencias de la 
instrucción práctica supervisada y las que ofrece el proceso de tesis. Es importante recalcar que los estudiantes argumentaron sobre el significado que tiene para ellos la instrucción práctica y el proceso de tesis.

Con respecto a las relaciones del contexto interior con el exterior, de manera notable abundan los pasajes y las instancias de los vínculos con Latinoamérica. Esto toma distancia del Paradigma Dialéctico de la Administración Crítica, en el sentido que nos impone la reflexión que el proceso administrativo local es parte del proyecto nacional global. Desde mi óptica primero integro el proyecto local autóctono y luego el nacional global.

\section{Recomendaciones a la luz de los hallazgos}

\section{A los directivos del PMTS}

\section{Liderazgo administrativo mediante planes articulados}

1. Desarrollar un proyecto de liderazgo administrativo endógeno autóctono, como proyecto educativo con los atributos de la comunidad universitaria de aprendices, donde la comunicación es abierta, todos aportan, reflexionan y co-construyen el conocimiento, cuida de sus miembros y valora sus aportaciones. Con la adopción de esta práctica el PMTS tomaría distancia del paradigma tradicional y entrarían de una manera dinámica en sintonía con el Paradigma Humanista Crítico.

2. El líder transformador según articulado por el resultado del análisis metaetnográfico desarrollado por Pielstick (1998) promoverá actividades endógenas para que los desarrollos epistemológicos vayan a la par con los desarrollos en prácticas didácticas. Además, provocará cambios en el personal administrativo y de la biblioteca involucrándolos en los procesos de participación y de desarrollo profesional continuo, en armonía con la misión, visión y el paradigma adoptado.

3. De igual modo, la comunidad universitaria de aprendices del PMTS promovería servicios para mujeres estudiantes con hijos pequeños, así como otros servicios de consejería y orientación al estudiantado más allá de los servicios que se le ofrecen en la actualidad. 
4. El PMTS podría utilizar como fuente permanente de producción de conocimiento las experiencias de instrucción práctica y de tesis. Estas dos instancias tienen la virtud de ser fuente para la integración de conocimiento, la creación y desarrollo de los talentos del estudiantado a la par con procesos de estudios o en líneas de investigación que adelante la Facultad, acorde con los planes de desarrollo profesional.

5. Utilizar de manera sistemática los aprendizajes de la Unidad de Proyectos Especiales y del Instituto de Política Social para el mantenimiento y revisión del currículo. Esto asegura la pertinencia del currículo a tono con la realidad social puertorriqueña; además, esto permite operacionalizar el acortamiento de la brecha entre el entendimiento de lo teórico y la práctica o la praxis, en congruencia con la realidad vivida.

6. La innovación tecnológica requiere de adoptar prácticas para encarar las competencias y las modificaciones constantes en los patrones de organización social. La recomendación concreta es la creación de la verdadera comunidad universitaria de aprendices en el PMTS.

\section{Liderazgo didáctico mediante planes articulados}

1. El liderazgo didáctico se articularía e integraría a los planes del liderazgo administrativo, se observarían las relaciones y la dinámica del liderazgo educativo como un todo integrado.

2. El liderazgo didáctico se ejercería con la identificación de necesidades de la Facultad y de las personas que participan en las experiencias de aprendizaje (Sparks y Hirsh, 1997), con el objetivo de desarrollar planes coherentes y estratégicos de desarrollo profesional y de los recursos humanos (Ramos, 1999) para armonizar el paradigma adoptado con la misión (reformulada) y visión del PMTS, de la Universidad y las prácticas del liderazgo educativo del Siglo XXI.

En la Figura 4 presento una representación esquematizada del cambio: hacia el Paradigma de la Comunidad Universitaria de Aprendices. Las líneas entrecortadas reflejan la constante relación de la cultura organizacional, como punto de partida que es impactada por e impacta a los contextos (Rodríguez Matos, 2001). La misma la componen los miembros de la comunidad de aprendices, la misión, la visión, los valores y los procesos de 
participación, toma de decisiones $\mathrm{y}$ de comunicación, como un todo integrado; a partir de un proyecto endógeno local, nacional y luego internacional, en adhesión a los marcos teóricos adoptados.

\section{Figura 4}

Representación esquematizada del Paradigma de las

Comunidades de Aprendices en el PMTS

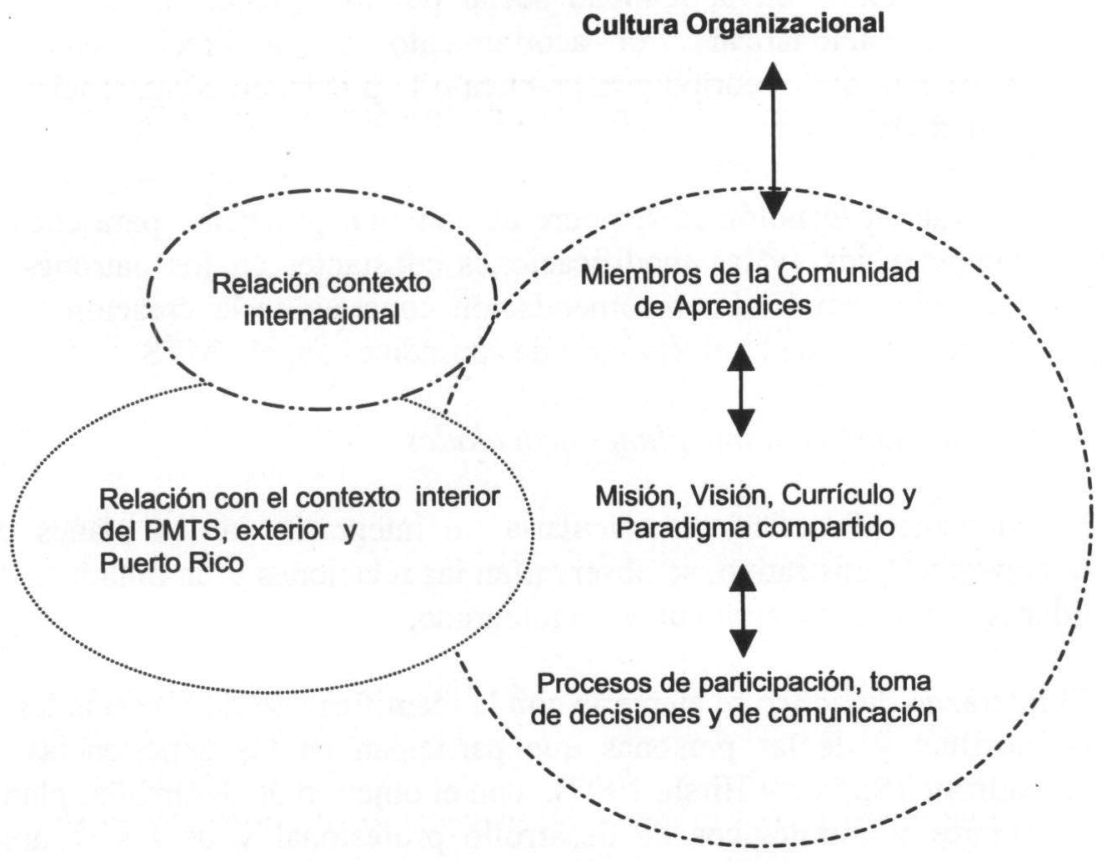

\section{A la Universidad de Puerto Rico, Recinto de Río Piedras}

Es conveniente resaltar que existen algunas situaciones que ameritan cambio y trasciende el alcance del PMTS. No obstante, considero que a partir del proyecto endógeno de la comunidad universitaria de aprendices del PMTS, es preciso alzar la voz con lo siguiente: 
1. Promover el desarrollo de planes de reestructuración profunda, que apunten a la atención de los asuntos administrativos, con agilidad y prontitud, esto, en contraposición a las tradicionales prácticas burocráticas.

2. Desarrollar una normativa acerca de la comunidad de aprendices enfocada a los procesos de liderazgo administrativo. Dado que, la normativa de comunidad de aprendices del Recinto de Río Piedras, está enfocada en los procesos de enseñanza aprendizaje. Esto deja al descubierto los procesos de liderazgo administrativo en el Recinto de Río Piedras.

3. Ofrecer servicios al estudiantado de cuido de niños, estacionamientos y a los que participan en los procesos de enseñanza aprendizaje, orientados a su desarrollo integral.

\section{Investigaciones futuras}

Dada la riqueza de información que ofrece el diseño etnográfico crítico, sugiero que se desarrollen estudios para documentar la concordancia del paradigma, la misión, la visión y el currículo en otras culturas organizacionales en la educación universitaria. Para finalizar, inspirada en Freire (1997), reconozco que el proceso educativo es uno inacabado. Por tanto, es útil insertar en la reflexión acerca de futuros estudios lo planteado por Freire (1997):

La raíz más profunda de la politicidad de la educación está en la propia educabilidad del ser humano, que se funde en su naturaleza inacabada y de la cual se volvió consciente. Inacabado y consciente de su inacabamiento, histórico, el ser humano se haría necesariamente un ser ético, un ser de opción, de decisión (p.106).

En otras palabras, este no es un estudio concluyente, más bien abre las oportunidades para continuar en la investigación crítica de la educación.

\section{Lo que la investigación ha hecho de mí}

Este estudio me permitió desarrollar una investigación microetnográfica en el PMTS, en todas sus fases de acuerdo a los marcos metodológicos y teóricos 
consultados. En todo momento los modelos metodológicos fueron guía para realizar este estudio en el contexto puertorriqueño.

Los actores, las voces, los documentos, lo espacial y lo físico del PMTS, me enseñaron y me confirmaron que la educación es un proceso dinámico en constante cambio. En esa misma dimensión los procesos deben verse como dinámicos. También me reconfirmaron que es necesario escudriñar para descubrir, para explorar y hacer la denuncia informada para provocar cambios. Las voces que no están audibles por las asimetrías en la relación de poder, me comprometieron a denunciar, más allá de lo que yo vi y escuché, lo testimoniaron con sus sentimientos expresados mediante gestos y también con sus silencios.

Esta investigación me sensibilizó más al entendimiento de los silencios, de la no participación, de los temores y de las incertidumbres que le produce a una cultura la llegada de una persona que no es parte de sus actividades cotidianas. Todo esto me llevó a buscar significados, a hacer interpretaciones para aumentar la conciencia crítica como educadora y como líder, particularmente como persona comprometida con la denuncia de las prácticas opresoras y provocar la transformación, a través de la educación que es lucha, es promesa, es futuro, es esperanza. Esto fue lo que hizo de mí este estudio.

\section{Referencias}

Argyris, Chris \& Schön, Donald (1996). Organizational learning. MA: Addison Wesley.

Bryk, Anthony \& Driscoll Mary (1988). The high school as community: Contextual influences and consequences for students and teachers. Madison, Wisconsin: National Center on Effective Secondary Schools, University of Wisconsin, ED 302 539. Recuperado el 28 de agosto de 2004, de http://web.ebscohost.com/ehost/detail.

Carspecken, Phil Francis (1996). Critical ethnography in educational research: A theoretical and practical guide. New York: Routlege.

Carspecken, P. F. (2002). The hidden history of praxis theory within critical ethnography and criticalism/postmodernism problematic. En Y. Zou y 
E.T. Trueba, (Eds.), Ethography and schools: Qualitative approaches to study of education (pp. 49-86). Boston, MA: Rowman \& Littlefield.

Castillo Ortiz, Alicia (2002). Prospectiva crítica del libro La estructura de la Administración crítica: Una interpretación dialéctica de Dr. José Miguel Rodríguez. Cuaderno de Investigación en la Educación, 17, 102-108.

Comisión internacional sobre la educación para el siglo XXI (1996). La educación encierra un tesoro. Madrid: Santillana - Ediciones UNESCO

Darling - Hammond, Linda (1996 march). The quiet revolution: Rethinking teacher development. Educational Leadership. 53 (6), 4-10.

Derrida, Jacques (1984). La filosofia como institución (A. Azurmendi, Trad.). Barcelona: Ediciones Juan Graníca.

Dempsey, Maria, Halton, Carmen, \& Murphy, Marian (2001). Reflective learning in social work education: Scafflolding the process. Social Work Education, 20 (6), 631-641. Recuperado el 15 de marzo de 2004, de http://www.questia.com.

Díaz Pérez, Yolanda (1978). Nature and focus of social work pracitce in Puerto Rico. Disertación doctoral no publicada, University of Denver, Colorado.

Dingwall, Robert \& Lewis, Philip (1983). The sociology of professions. London: Mcmillan.

Drucker, Peter (1997). The organization of the future. San Francisco, CA.: Jossey Bass.

DuFour, Richard, \& Eaker, Robert (1998). Professional learning communities at work: Best practices for enhancing student achievement. Bloomington, IN. National Educational Services.

Elmore, Richard (1995 december). Structural reform and educational practice. Educational Research, 24(9). Recuperado el 20 de octubre de 2006, www.jstor.org/journals/ 0013189X.html 
Fasoli, Héctor (2003). Enseñanza e investigación con vocación de servicio.

Recuperado el 27 de diciembre de 2004, de

http://www.uca.edu.ar/esp/sec-

pigpp/esp/page.php?subsec=estudios\&page=revista/tp9.pdf

Freire, Paulo (1997). Pedagogía de la autonomía. Mexico: Siglo xxi.

Fullan, Michael (2000). The stories of education reform. Phi Delta Kappan, 81 (8), 581-584. Recuperado el 20 de octubre de 2006, de http://www.pdkintl.org/kappan/kful0004.htm

García Fernández, Nicanor (2002). Las comunidades de aprendizaje: Sistemas de trabajo con las TICs en el sistema educativo y en la formación de profesionales. Recuperado el 3 de mayo de 2003, de http:scholar.google.com.pr/scholar.

Hipp, Kristine Kiefer, Stoll, Louise, Bolam, Ray, Wallace, Mike, McMahon, Agnes, Thomas, Sally, \& otros. (2003, abril). An international perspective on the development of learning communities. Documento presentado en la reunión anual de la American Educational Research Association, Chicago, IL, EE.UU. Recuperado el 14 de enero de 2005, de

http://eric.ed.gov/ERICWebPortal/Home.ED478734

Kincheole, Joe L., \& McLaren, Peter (2002). Rethinking critical theory and qualitative research. En Y. Zou y E.T. Trueba, (Eds.), Ethography and schools: Qualitative approaches to study of education (pp. 87-138). MD: Rowman \& Littlefield. ED435342

Knowles, Malcolm (1980). The modern practice of adult education. New York: University Press

Lizardi, María Milagros (1983). Origins and development of social work education in Puerto Rico. Disertación doctoral no publicada, Tulane University, New Orleans.

Lucca Irizarry, Nydia \& Berríos Rivera, Reinaldo (2003). Investigación cualitativa en educación y ciencias sociales. San Juan, PR: Publicaciones Puertorriqueñas. 
Malaclaza, Susana Leonor (2003). Desde el imaginario social del siglo XXI: Repensar el Trabajo Social. Buenos Aires: Espacio Social.

Manfredo Plicet, Ursula (2004). En búsqueda de la comunidad de aprendices: Un estudio etnográfico el Programa de Maestría de la Escuela Graduada de Trabajo Social de la Universidad de Puerto Rico. Trabajo de investigación doctoral para el curso Investigación Etnográfica. Manuscrito no publicado, Universidad de Puerto Rico, Río Piedras, PR.

Malott, María E. (1998). Misión universitaria: calidad de vida a través de la educación superior. Agenda académica, 5 (2). Recuperado el 27 de diciembre de 2004, de http://www.revele.com.ve /pdf/agenda/vol5n2/pag3.pdf\#search=\%22Malott.

McLaughin, Milbrey, \& Talbert, Joan (2001). Professional Communities and the Work of High School Teaching. Chicago: University of Chicago Press.

Mitchell, Coral, \& Sackney, Lany (2000). Professional improvement: Building capacity for a teaching community. Lisse, NL: Swets \& Zeitlinger.

Morales Santiago, Gamaliel (2003). Hacia una concepción de liderazgo crítico en la administración educativa. Disertación doctoral inédita. Universidad de Puerto Rico, Recinto de Río Piedras.

Morrow, Raymond Allan \& Torres, Carlos Alberto (2002). Las teorías de la reproducción social y cultural. España: Editorial Popular.

Newmann, Fred M., \& Associates (1996). Authentic achievement: Restructuring schools for intellectual quality. San Francisco: Jossey Bassey

Ortega y Gasett, José (1936). Misión de la Universidad: y otros ensayos afines. Madrid: Ediciones Revista Occidente. 
Pielstick, C.Dean (1998). The transforming leader: A meta-ethnographic analysis. Community College Review 26 (3). Recuperado el 14 de febrero de 2004, de http://www.questia.com.

Ramos Rodríguez, Isabel (1999). Desarrollo profesional de los educadores $y$ de las educadoras. San Juan, PR: Publicaciones Puertorriqueñas.

Raywid, Mary Anne (1993). Community: An alternative school accomplishment. In public schools that work: Creating community. En G. A. Smith (Ed.) New York: Routledge.

Rodríguez Matos, José Miguel (2001). La estructura de la administración crítica: Una interpretación dialéctica. San Juan, PR: Asociación Puertorriqueña de Profesores Universitarios.

Rojas-Drumnond, Sylvia (1998). Creando comunidades de aprendizaje en las escuelas primarias de México. Recuperado el 3 de mayo de 2003, de http://educacion. jalisco.gob.mx/consulta/educar/09/9sylviar.html

Royal, Mark A., \& Rossi, Robert J. (1997). Schools as communities. ED405641. Recuperado el 26 de agosto de 2004, http://ericfacility.net/ericdigests/ed405641.html

Senge, Peter M. (1990). The fifth discipline: The art and practice of the learning organization. New York: Currency Doubleday.

Solís Jordán, José (2005). Hacia la investigación crítica en la educación: Insurgencias cualitativas. San Juan, PR: Publicaciones Puertorriqueñas.

Sparks, Dennis \& Hirsh, Stephanie (1997). A new vision for staff development. Alexnadia: VA, Association for Supervision and Curriculum Development.

Tedesco, Juan Carlos (1985). Reproduccionismo educativo y sectores populares en América Latina. Sao-Paulo: Cortés Editora.

Thomas, Jim (1993). Doing critical ethnography. Newbury Park, CA: Sage University Paper. 
Toole, James C., \& Louis, Karen Seashore (2002). The role of professional learning communities in international education. En K. Leithwood and P. Hallinger (Eds). Second International Handbook of Educational Leadership and Administration. London: Kluver.

Universidad de Puerto Rico. (1999). Hacia una comunidad de aprendizaje: Reflexiones y recomendaciones en torno a los procesos de enseñanzaaprendizaje en el Recinto de Río Piedras, Universidad de Puerto Rico. Centro de Excelencia Académica, Decanato de Asuntos Académicos: Autor.

Universidad de Puerto Rico. (2002). Self study report for the reaffirmation of accreditation submitted to the Commission of Accreditation on Social Work Education, Río Piedras Campus, PR: Author.

Universidad de Puerto Rico. (2005). Propuesta para la revisión del Programa de Maestría en Trabajo Social de la Facultad de Ciencias Sociales del Recinto de Río Piedras de la Universidad de Puerto Rico. (Vol. I y II.) Recinto de Río Piedras, PR: Autor.

Villalobos Torres, Elvia Marveya (2000). Formación docente en estrategias de aprendizaje. Revista Panamericana de Pedagogía. Recuperado el 6 de septiembre de 2003, de http://web.ebscohost.com/ehost/pdf. 
